\title{
COMPARING [C II], Hi, AND CO DYNAMICS OF NEARBY GALAXIES
}

\author{
W. J. G. De Blok ${ }^{1,2,3}$, F. Walter ${ }^{4}$, J.-D. T. Smith $^{5}$, R. Herrera-Camus ${ }^{6,7}$, A. D. Bolatto ${ }^{6,7}$, M. A. Requena-Torres ${ }^{8}$, \\ A. F. Crocker ${ }^{5,9}$, K. V. Croxall ${ }^{10}$, R. C. Kennicutt ${ }^{11}$, J. Koda ${ }^{12}$, L. Armus ${ }^{13}$, M. Boquien ${ }^{14}$, D. Dale ${ }^{15}$, \\ K. KRECKEL ${ }^{4}$, AND S. MEIDT ${ }^{4}$ \\ ${ }^{1}$ Netherlands Institute for Radio Astronomy (ASTRON), Postbus 2, 7990 AA Dwingeloo, The Netherlands \\ ${ }^{2}$ Astrophysics, Cosmology and Gravity Centre, Univ. of Cape Town, Private Bag X3, Rondebosch 7701, South Africa \\ ${ }^{3}$ Kapteyn Astronomical Institute, University of Groningen, P.O. Box 800, 9700 AV Groningen, The Netherlands \\ ${ }^{4}$ Max-Planck Institut für Astronomie, Königstuhl 17, D-69117, Heidelberg, Germany \\ ${ }^{5}$ Department of Physics and Astronomy, University of Toledo, Toledo, OH 43606, USA \\ ${ }^{6}$ Department of Astronomy, University of Maryland, College Park, MD 20742, USA \\ ${ }^{7}$ Laboratory for Millimeter-Wave Astronomy, University of Maryland, College Park, MD 20742, USA \\ ${ }^{8}$ Space Telescope Science Institute, 3700 San Martin Dr., Baltimore, MD 21218, USA \\ ${ }^{9}$ Department of Physics, Reed College, Portland, OR 97202, USA \\ ${ }^{10}$ Department of Astronomy, The Ohio State University, 4051 McPherson Laboratory, 140 West 18th Avenue, Columbus, OH 43210, USA \\ ${ }^{11}$ Institute of Astronomy, University of Cambridge, Madingley Road, Cambridge CB3 OHA, UK \\ ${ }^{12}$ Department of Physics and Astronomy, Stony Brook University, Stony Brook, NY 11794-3800, USA \\ ${ }^{13}$ Spitzer Science Center, California Institute of Technology, Pasadena, CA 91125, USA \\ ${ }^{14}$ Universidad de Antofagasta, Unidad de Astronomía, Avenida Angamos 601, 02800 Antofagasta, Chile \\ ${ }^{15}$ Department of Physics \& Astronomy, University of Wyoming, WY 82071, USA \\ Received 2015 December 3; accepted 2016 April 28; published 2016 August 4
}

\begin{abstract}
The $\mathrm{H}_{\mathrm{I}}$ and $\mathrm{CO}$ components of the interstellar medium (ISM) are usually used to derive the dynamical mass $M_{\mathrm{dyn}}$ of nearby galaxies. Both components become too faint to be used as a tracer in observations of high-redshift galaxies. In those cases, the $158 \mu \mathrm{m}$ line of atomic carbon ([C $\mathrm{CI}]$ ) may be the only way to derive $M_{\mathrm{dyn}}$. As the distribution and kinematics of the ISM tracer affects the determination of $M_{\mathrm{dyn}}$, it is important to quantify the relative distributions of $\mathrm{H}_{\mathrm{I}}$, $\mathrm{CO}$, and $[\mathrm{C} \mathrm{II}] . \mathrm{H} \mathrm{I}$ and $\mathrm{CO}$ are well-characterized observationally, however, for $[\mathrm{C} \mathrm{II}]$ only very few measurements exist. Here we compare observations of $\mathrm{CO}, \mathrm{H}_{\mathrm{I}}$, and $[\mathrm{C} \mathrm{II}]$ emission of a sample of nearby galaxies, drawn from the HERACLES, THINGS, and KINGFISH surveys. We find that within $R_{25}$, the average [C $\mathrm{II}$ exponential radial profile is slightly shallower than that of the $\mathrm{CO}$, but much steeper than the $\mathrm{H}$ I distribution. This is also reflected in the integrated spectrum ("global profile"), where the [C $\mathrm{CI}]$ spectrum looks more like that of the $\mathrm{CO}$ than that of the $\mathrm{H} \mathrm{I}$. For one galaxy, a spectrally resolved comparison of integrated spectra was possible; other comparisons were limited by the intrinsic linewidths of the galaxies and the coarse velocity resolution of the $[\mathrm{C}$ II] data. Using high-spectral-resolution SOFIA $\left[\mathrm{C}_{\mathrm{II}}\right]$ data of a number of star forming regions in two nearby galaxies, we find that their $\left[\mathrm{C}_{\mathrm{II}}\right]$ linewidths agree better with those of the CO than the $\mathrm{H}_{\mathrm{I}}$. As the radial extent of a given ISM tracer is a key input in deriving $M_{\mathrm{dyn}}$ from spatially unresolved data, we conclude that the relevant length-scale to use in determining $M_{\mathrm{dyn}}$ based on [C II] data, is that of the well-characterized $\mathrm{CO}$ distribution. This length scale is similar to that of the optical disk.
\end{abstract}

Key words: galaxies: fundamental parameters - galaxies: ISM - galaxies: kinematics and dynamics - radio lines: galaxies - submillimeter: galaxies

Supporting material: figure sets

\section{INTRODUCTION}

Observations of the gas component of galaxies yield important information on their dynamics. For example, through the use of rotation curves, detailed knowledge of the distribution of dark matter in galaxies has become available. Where rotating galaxies are not spatially resolved, their global profile (or integrated spectrum, i.e., the distribution of spatially integrated flux per velocity interval as a function of apparent radial Doppler velocity) still provides information on dynamical masses $M_{\text {dyn }} \propto V^{2} R / G$, where $V$ is the rotation velocity at radius $R$, and $G$ is the gravitational constant.

The dynamical mass therefore measures the total mass content of a galaxy within a certain radius $R$, including that of the dark matter. The dark matter distribution does, however, extend significantly beyond that of the visible matter, and the total dynamical mass (i.e., as measured at the virial radius) cannot be determined in the absence of visible tracers at these large radii.
Dynamical mass measurements using the visible galaxy are therefore always lower limits and depend on the radius at which the measurement is made. A consistent choice for the radius $R$ at which $M_{\mathrm{dyn}}$ is measured is critically important for comparisons between different galaxies, but also for measurements using different tracers in the same galaxy. For example, in local disk galaxies the $\mathrm{H}$ I disk typically extends significantly beyond the main stellar disk. Using the outer radii of either the stellar or the H I disk will result in two different values of $M_{\mathrm{dyn}}$. These can only be consistently compared once the typical distribution of each of the tracers is taken into account.

For low-redshift galaxies, both atomic neutral hydrogen $\left(\mathrm{H}_{\mathrm{I}}\right)$ and $\mathrm{H} \alpha$ are routinely used to study galaxy dynamics. $\mathrm{H} \alpha$ has the advantage that it can be observed at high spatial resolution, but it is generally confined to the optical disks of galaxies. It can also be more prone to the effects of extinction as well as random motions due to, e.g., massive star formation.

$\mathrm{H}_{\mathrm{I}}$ is easily observable in the $21 \mathrm{~cm}$ radio line, constituting the main gas component in most disk galaxies. It usually extends 
beyond the optical disk, enabling studies of the dynamics out to large radii. The $\mathrm{H}$ I tends to have a high disk surface covering factor, and has a fairly constant surface density over most of its radial extent. Many studies of the kinematics of disk galaxies thus rely heavily on $\mathrm{H}$ i observations.

The $\mathrm{H}_{\mathrm{I}}$ line has a disadvantage, though, in that the nature of the transition that gives rise to it makes it intrinsically faint. This makes it still challenging to observe $\mathrm{HI}$ at redshifts $z \gtrsim 0.2$ (e.g., Verheijen et al. 2007). Future telescopes, such as the Square Kilometre Array, should make these observations more feasible, but even with such large facilities, observations of $\mathrm{H}$ in galaxies at $z \sim 1$ and beyond will not be trivial (see, e.g., Blyth et al. 2015).

Observations of the kinematics of galaxies at high redshift are also being obtained by observing redshifted optical emission lines such as $\mathrm{H} \alpha$ using Integral Field Unit instruments. Compared to low-redshift observations an added uncertainty in the interpretation of these kinematics is the importance of noncircular motions (due to turbulence, winds, and/or streaming motions in non-axisymmetric potentials) in very actively starforming galaxies. It is generally expected that at higher redshifts these are more important. Some early observations of the kinematics of disk galaxies at high redshift are presented in, e.g., Vogt et al. (1996), Förster Schreiber et al. (2006) and Genzel et al. (2006).

At higher redshifts, $\mathrm{CO}$ has also been used as a tracer for galaxy dynamics. $\mathrm{CO}$ lines are intrinsically much brighter than the $\mathrm{H}$ I line, but the $\mathrm{CO}$ distribution is more compact and, at least at low redshift, tied to that of the stellar component (e.g., Regan et al. 2006; Leroy et al. 2008, 2013 and Schruba et al. 2011). Despite the more compact extent it is still a useful tool to study galaxy dynamics.

However, even $\mathrm{CO}$ detections are challenging at very high $(z>5)$ redshifts. This is due to a combination of different factors. First, low- $J \mathrm{CO}$ transitions, that trace the bulk of the molecular gas, are shifted to long wavelengths that are difficult to access with current facilities (e.g., Carilli \& Walter 2013). Second, the cosmic microwave background (which has a temperature proportional to $1+z$ ) sets the effective bottom temperature of the interstellar medium (ISM) of any galaxy which in turn makes it difficult to detect gas at intrinsically low temperatures (da Cunha et al. 2013). Lastly, if the metallicities are sufficiently low, studies at low redshift have shown that the $\mathrm{CO}$ emission is decreased for a given $\mathrm{H}_{2}$ mass (e.g., Wilson 1995; Schruba et al. 2012). This will also affect measurements in main sequence galaxies at very high redshift, where metallicities are depressed (e.g., Genzel et al. 2012).

An alternative gas tracer is the $158 \mu \mathrm{m}$ line of atomic carbon, $\mathrm{C}^{+}$. This is one of the main cooling lines of the ISM, and is typically the brightest line (for exceptions see, e.g., Madden et al. 2011; Cormier et al. 2015).

[C II] is also well-known to undergo relatively large changes in its fractional cooling power relative to bolometric infrared luminosity (TIR)—-the so-called "cooling line deficit" (e.g., Luhman et al. 2003, J.-D. T. Smith et al. 2016, in preparation). At high star formation rate (SFR) surface density, as is found in the local universe at the highest infrared luminosities, [C II]/ TIR can drop significantly. At higher redshift, however, luminous systems are often extended and can have MilkyWay like or even higher [C II]/TIR ratios (Brisbin et al. 2015).

On the other hand, Cormier et al. (2015) and Madden et al. (2011) show that in low-metallicity environments $\mathrm{C}^{+}$remains bright with respect to $\mathrm{CO}$ and FIR emission and increases in relative power as metallicity declines in resolved nearby galaxies (J.-D. T. Smith et al. 2016, in preparation). $\mathrm{C}^{+}$is distributed throughout the ISM and as it can be excited by collisions with electrons, $\mathrm{H}_{\mathrm{I}}$ and $\mathrm{H}_{2}$, it is expected to trace most of the phases of the ionized, atomic and molecular ISM (e.g., Pineda et al. 2013).

The downside of [C $\mathrm{II}]$ is that its rest-frequency is very high: at $\sim 1900 \mathrm{GHz}(158 \mu \mathrm{m})$ observations of low-redshift galaxies from the ground are impossible. However, moving to higher redshifts means the frequency of the line shifts into regions of the spectrum that are observable from the ground. With the advent of ALMA (particularly in Bands 8, 9, 10) it is therefore expected that observations of $\mathrm{C}^{+}$can be routinely done for galaxies at $z>1$ (see e.g., Maiolino et al. 2005; Venemans et al. 2012; Walter et al. 2012), and in principle out to extreme redshifts $(z \sim 20)$.

$\mathrm{C}^{+}$observations are therefore likely to become an important tool in determining dynamical masses and other kinematical properties of galaxies at high redshifts. A disadvantage of the high redshifts is that detailed spatial information will not be available since galaxies will be hard to resolve. As an example, an 0 . 5 beam will measure $\sim 3-4 \mathrm{kpc}$ beyond $z \sim 1$. Surface brightness sensitivity considerations make it unlikely that significantly higher resolutions can be achieved with current instruments unless significant amounts of observing time (e.g., $>10^{h}$ with ALMA) are invested.

Galaxies at these redshifts will therefore typically only be resolved by a small number of beams along their semimajor axis. This means that determinations of quantities such as the dynamical mass of systems will have to be based on global velocity profiles (integrated spectra). Determining a dynamical mass using this method requires a velocity width and a representative radius. The former is readily determined from the profile, but the latter is generally unknown. In practice, a multiple of the optical radius or some other measure of the expected extent of the tracer is used. This choice is important in, e.g., proper comparisons between the baryonic and the dynamical mass. Choosing a radius that is not appropriate for the tracer used can lead to severe over- or under-estimates of the dynamical mass and therefore skewed comparisons. See de Blok \& Walter (2014) for further discussion on this.

A spatially integrated spectrum, or "global profile" as it is usually called in radio-astronomy, is a general observable of galaxies, independent of their dynamical state. For the case of a rotating galaxy, with gas on circular orbits, the global profile is defined by the rotation curve and the gas tracer distribution. The rotation curve depends on the total mass distribution within the galaxy (including stars and dark matter), and the rotation velocity as a function of radius therefore does not depend on the type of tracer used to measure the velocities. The radial mass surface density distributions of the tracers can, however, differ. de Blok \& Walter (2014) demonstrate how the shape of the radial distribution of the tracer has an impact on the shape and velocity width of the global profile. In other words, determining the integrated spectrum of a galaxy in either $\mathrm{H}_{\mathrm{i}}$ or $\mathrm{CO}$ will likely not lead to identical profiles (see Frank et al. 2015 for examples). In interpreting any measurement from a global profile it is therefore important to know what tracer was used and what its likely radial distribution is. This directly affects any interpretation of the Tully-Fisher relation (Tully \& Fisher 1977). Several papers compare the 
observed linewidths as measured for different tracers within this context. For example, Mathewson et al. (1992) compare $\mathrm{H} \alpha$ and $\mathrm{H}$ i linewidths. Observed $\mathrm{CO}$ and $\mathrm{H}$ Ilinewidths are compared in, e.g., Sofue (1992), Dickey \& Kazes (1992), Schoniger \& Sofue (1994) and Frank et al. (2015). de Blok \& Walter (2014) use simple models to quantify the effects of the radial distributions of various tracers on linewidths and the Tully-Fisher relation.

Here we discuss and compare radial profiles and spatially integrated spectra as derived from [C $\left.\mathrm{C}_{\mathrm{II}}\right]$ observations. For this we will use spatially resolved [C II] data of nearby galaxies and make a direct comparison between the overall distributions and kinematics of the [C II], $\mathrm{CO}$ and $\mathrm{H}_{\mathrm{I}}$. The goal is to test whether global profiles derived from [C $\mathrm{II}]$ measurements resemble global profiles derived from $\mathrm{CO}$ data (with an inherently compact tracer distribution) or from $\mathrm{H}$ i data (where the tracer is much more extended). We use observations of individual starforming regions to also extend the comparison to velocity dispersions.

In Section 2 we present the sample and the data products that we use in our analysis. Section 3 compares the radial profiles of the gas tracers. In Section 4 we discuss the kinematics of the three gas tracers and construct spatially integrated spectra. Section 5 presents measurements of the spectra of individual star forming regions. We summarize our findings in Section 6.

\section{SAMPLE AND DATA}

The only set of galaxies where detailed, resolved information is available for all three tracers, $\mathrm{CO}, \mathrm{H}_{\mathrm{I}}$ and $[\mathrm{C} \mathrm{II}]$, is the overlap of the THINGS (H i; Walter et al. 2008), HERACLES (CO $J=2 \rightarrow 1$; Leroy et al. 2009), and KINGFISH ([C II] 158 $\mu \mathrm{m}$; Kennicutt et al. 2011) samples. Galaxies in these samples were selected to mostly be disk galaxies dominated by rotation and without strong bulge or bar features. By design, a significant number of galaxies is in common to these three surveys and can thus be used for a direct comparison of the detailed $\mathrm{HI}, \mathrm{CO}$ and $[\mathrm{C} \mathrm{II}]$ distribution and kinematics. For many of the galaxies in these samples detailed studies of the kinematics in the $\mathrm{H}$ I and $\mathrm{CO}$ are already available (de Blok et al. 2008; Frank et al. 2015).

The main criterion for our sample selection was the availability of high signal-to-noise and extended $\mathrm{H}$ I, $\mathrm{CO}$ and $[\mathrm{C}$ II] data for each of the galaxies. This resulted in a final sample of 10 galaxies. Table 1 lists this sample, along with some basic properties. ${ }^{16}$

A few of the galaxies have very low inclinations which would make them less suitable for dynamical mass measurements (due to the $\sin i$ correction that needs to be applied to correct the line of sight velocities); however, we include them here as the emphasis of this paper is on a comparison of observed radial mass distributions and integrated spectra and we do not try to calculate the actual dynamical masses.

In the end, the presence of a clear $\mathrm{CO}$ detection turned out to be the most stringent criterion. For this reason our sample mostly consists of spiral galaxies. As described below, all three data sets have a similar angular resolution of $\sim 12^{\prime \prime}$. For a

\footnotetext{
${ }^{16}$ NGC 7331 and NGC 3198 would have qualified as well. However, for these two galaxies the KINGFISH data were taken along the minor axis, making them less suited for an analysis of the dynamics. NGC 925 and NGC 2841 also have detections in all three tracers; however, the $\mathrm{CO}$ emission is too faint to provide useful constraints. Finally, NGC 3077 was not added to the sample as it is not a regularly rotating disk galaxy.
}

Table 1

Properties of Sample Galaxies

\begin{tabular}{lccccc}
\hline \hline Name & $\begin{array}{c}D \\
(\mathrm{Mpc})\end{array}$ & $\begin{array}{c}M_{B} \\
(\mathrm{mag})\end{array}$ & $\begin{array}{c}i \\
\left({ }^{\circ}\right)\end{array}$ & $\begin{array}{c}\log D_{25} \\
(\log 0 ! 1)\end{array}$ & $\begin{array}{c}\text { Metal. } \\
(12+\log (\mathrm{O} / \mathrm{H})) \\
(1)\end{array}$ \\
\hline NGC 0628 & 7.3 & -19.97 & 7 & 1.99 & 8.33 \\
NGC 2976 & 3.6 & -17.78 & 65 & 1.86 & 8.30 \\
NGC 3184 & 11.1 & -19.92 & 16 & 1.87 & 8.48 \\
NGC 3351 & 10.1 & -19.88 & 41 & 1.86 & 8.60 \\
NGC 3521 & 10.7 & -20.94 & 73 & 1.92 & 8.36 \\
NGC 3627 & 9.3 & -20.74 & 62 & 2.01 & 8.43 \\
NGC 4736 & 4.7 & -19.80 & 41 & 1.89 & 8.31 \\
NGC 5055 & 10.1 & -21.12 & 59 & 2.07 & 8.42 \\
NGC 5457 & 7.4 & -21.05 & $22^{\mathrm{a}}$ & 2.38 & 8.52 \\
NGC 6946 & 5.9 & -20.61 & 33 & 2.06 & 8.40 \\
\hline
\end{tabular}

Notes. (1) Name of galaxy (2) Distance (Mpc). (3) Absolute $B$-band magnitude (4) inclination (degrees) (5) $\log D_{25}(\log 0$ '.1) (6) Metallicity. Quantities in columns (2)-(6) are from Walter et al. (2008) and Kennicutt et al. (2003).

${ }^{a}$ Inclination from Colombo et al. (2014).

galaxy at the typical distance in our sample $(D \sim 8 \mathrm{Mpc})$, this corresponds to a linear resolution of $\sim 0.5 \mathrm{kpc}$.

We show the integrated emission (zeroth moment) maps and the velocity fields (first moment) for each galaxy in Figure 1. In all panels, the $\mathrm{H}$ idata are shown in grayscale in the background, with $\mathrm{H}$ I, $\mathrm{CO}$ and $[\mathrm{C} \mathrm{II}]$ data overlaid as contours. Both the $\mathrm{H}$ i and $\mathrm{CO}$ observations cover the entire galaxy out to at least $R_{25}$, but the [C $\mathrm{II}$ ] observations are typically restricted to a smaller region, as indicated in the figures.

\section{1. [C II] Data}

The [C $\mathrm{II}] 158 \mu \mathrm{m}$ data were obtained as part of the KINGFISH survey (Kennicutt et al. 2011). The observations were done with the PACS instrument (Poglitsch et al. 2010) on board Herschel (Pilbratt et al. 2010). For a detailed description of the data reduction we refer to Croxall et al. (2013) and K. V. Croxall et al. (2016, in preparation).

Most of the data consists of strip maps, usually (though not always) along the major axis of the galaxy. In our sample selection we have selected only galaxies where the orientation of the strip is close to or along the major axis. The orientation is defined by that of the SINGS observations with the Spitzer Infra-Red Spectrograph instrument's Long-Low module of strips covering the central regions of the galaxies (see Kennicutt et al. 2003; Smith et al. 2007). For a number of galaxies extra-nuclear pointings of approximately $1 \operatorname{arcmin}^{2}$ in size were also obtained, which are combined with the corresponding strip map.

The angular resolution of the $[\mathrm{C}$ II $]$ data is $12^{\prime \prime}$. A pixel size of 2 ". 6 was adopted. The spectral resolution is $\sim 239 \mathrm{~km} \mathrm{~s}^{-1}$. Here we use the $[\mathrm{C}$ II] data cubes, integrated intensity maps (zeroth moment) and the velocity fields (first moment) that are part of the KINGFISH DR3 data products. ${ }^{17}$ Derivation of these maps is described in K. V. Croxall et al. (2016, in preparation).

For two of our galaxies (NGC 5457 and NGC 6946), high spectral-resolution SOFIA observations of the [C II] in individual star-forming regions are available which are discussed in Section 2.6.

\footnotetext{
$17 \mathrm{ftp}$ //hsa.esac.esa.int and navigate to LEGACY PRODUCTS/UPDP/ KINGFISH-DR3/.
} 


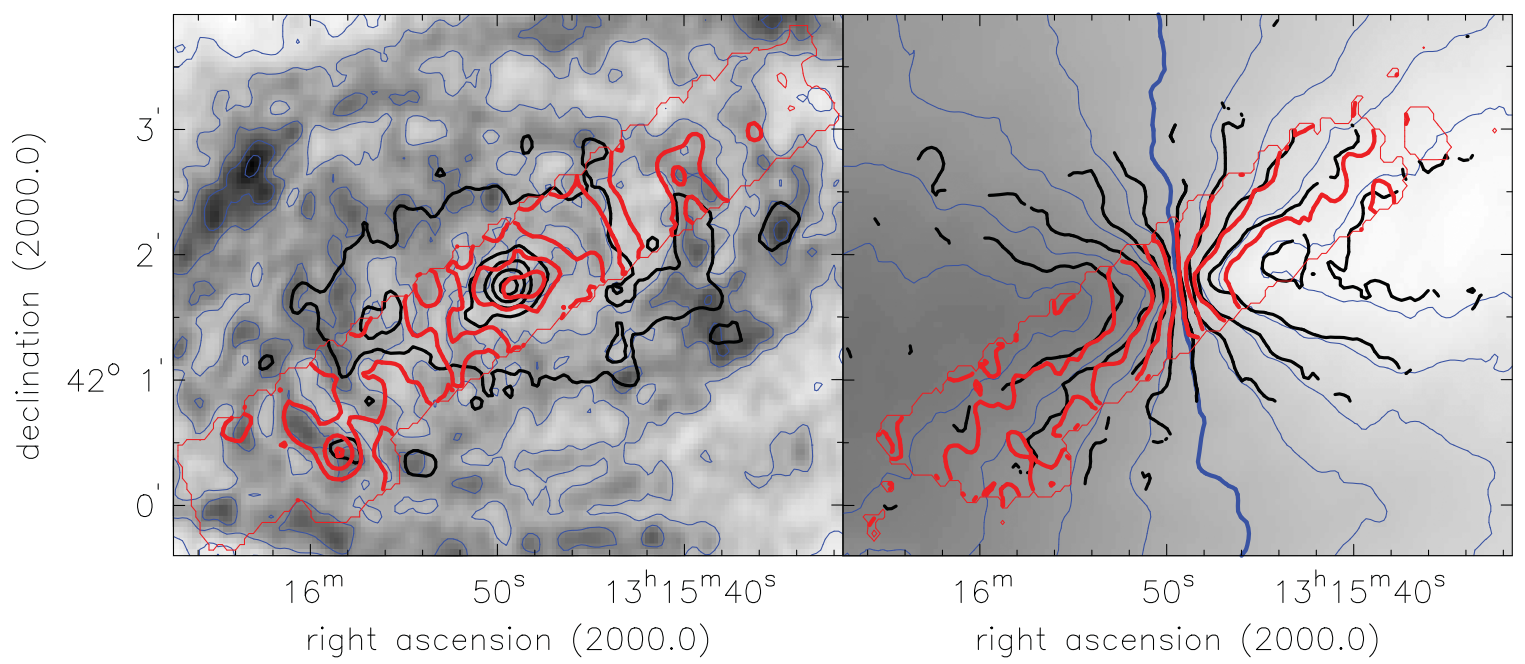

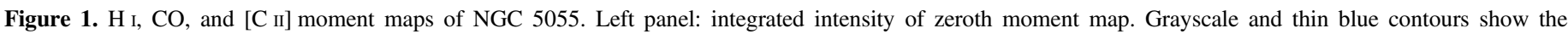

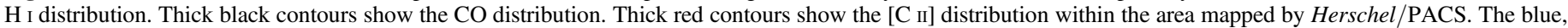

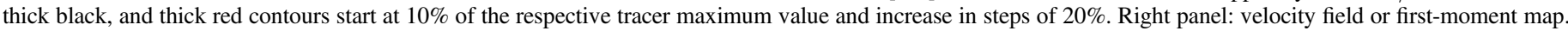

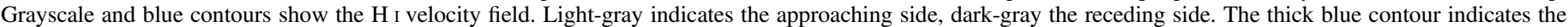

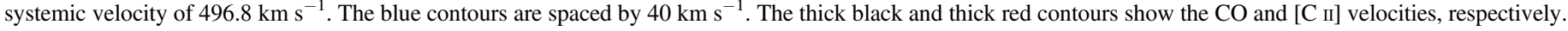

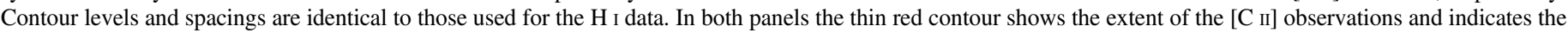
masks used for the $\mathrm{CO}$ and $\mathrm{H}$ i observations.

(The complete figure set (10 images) is available.)

\subsection{CO Data}

We use the $\mathrm{CO}(J=2 \rightarrow 1)$ data obtained as part of the HERACLES survey (Leroy et al. 2009). The angular resolution of the HERACLES data is $13^{\prime \prime}$ by construction, the velocity spacing is $5.2 \mathrm{~km} \mathrm{~s}^{-1}$. We use the data cubes, the integrated column density (zeroth moment) maps and the intensityweighted mean velocity fields (first moment) that are part of the HERACLES data release. ${ }^{18}$ We refer to Leroy et al. (2009) for a further description of the properties and derivations of these maps. The velocities in the HERACLES data were corrected from LSR to heliocentric velocities.

\subsection{H I Data}

For the H I data we use the datacubes, the integrated column density (zeroth moment) maps and the intensity-weighted mean velocity fields (first moment) from the THINGS survey ${ }^{19}$, where we choose the maps derived from the natural-weighted, residual-scaled data cubes.

The THINGS observations of the galaxies in this paper have an angular resolution of $\sim 10^{\prime \prime}$, the velocity spacing is 2.6 or $5.2 \mathrm{~km} \mathrm{~s}^{-1}$. We refer to Walter et al. (2008) for a description of the properties and derivation of the individual maps and data cubes.

\subsection{Resolutions and Spatial Coverage}

As can be seen from the brief descriptions above, the angular resolutions of the $\mathrm{CO}, \mathrm{H}_{\mathrm{I}}$ and $\left[\mathrm{C}_{\mathrm{II}}\right]$ data are similar. We will therefore use and analyze these data sets at their native angular resolutions, without any additional spatial smoothing. The situation is different with the velocity resolutions. The $\mathrm{CO}$ and $\mathrm{H}$ I data have similar resolutions, in contrast with the $[\mathrm{C}$ II] data.

\footnotetext{
18 http://www.mpia.de/HERACLES

19 http://www.mpia.de/THINGS
}

The effects of the differences in spectral resolutions will be discussed in Section 4. The [C II] data consist mainly of strip maps with restricted areal coverage. In our further analysis we therefore only consider those areas in the $\mathrm{CO}$ and $\mathrm{H}$ i data that are also covered by the $[\mathrm{C} \mathrm{II}]$ observations.

\subsection{Comparison of Velocity Fields}

We compare the [C II], $\mathrm{H}$ I and $\mathrm{CO}$ velocities on a pixel-bypixel basis. The $\mathrm{CO}$ and $\mathrm{H}$ ivelocity maps were regridded to have the same grid spacings as the $[\mathrm{C}$ II] data. The [C $\mathrm{II}]$ velocity fields contain a small number of noisy pixels, mainly at the edges of the observed emission where the [C $\mathrm{CI}]$ intensity is low. These were removed by applying an intensity cut: velocity field pixels where the corresponding intensity in the integrated [C II] map was below $8 \cdot 10^{-9} \mathrm{~W} \mathrm{~m}^{-2} \mathrm{sr}^{-1}$ were blanked, as were the corresponding pixels in the $\mathrm{CO}$ and $\mathrm{H}$ I velocity fields.

In the left panel of Figure 2 we plot the measured velocities (vertical axis: $\mathrm{CO}$ and [C II], horizontal axis: $\mathrm{H}_{\mathrm{I}}$ ) for each pixel. Overall there is excellent agreement between the velocities, as expected for rotating gas disks in which all components are embedded in the same gravitational potential. However, in some cases, small offsets are seen.

Therefore, in the right panel of Figure 2 we plot the difference of the $\mathrm{CO}$ and $[\mathrm{C} \mathrm{II}]$ velocities (vertical axis) as a function of the difference of the $\mathrm{H}_{\mathrm{I}}$ and $\left[\mathrm{C}_{\mathrm{II}}\right]$ velocities (horizontal axis) for each pixel. The small offsets between the $\left[\mathrm{C}_{\mathrm{II}}\right]$ velocities on the one hand, and the $\mathrm{CO}$ and $\mathrm{H}_{\mathrm{I}}$ velocities on the other hand, are also seen clearly here. These systematic offsets of the [C $\mathrm{II}]$ velocities, which are up to $\sim 20 \mathrm{~km} \mathrm{~s}^{-1}$ on average (and usually much less), are small compared to the native velocity resolution of $\sim 239$ $\mathrm{km} \mathrm{s}^{-1}$ of the [ $\left.\mathrm{C}_{\mathrm{II}}\right]$ data. We consider it unlikely that these offsets represent true, physical velocity differences between gas components. Rather, they are likely the result of systematics in the $[\mathrm{C}$ II] data reduction process combined with the low velocity resolution of the $[\mathrm{C}$ II] profiles. 

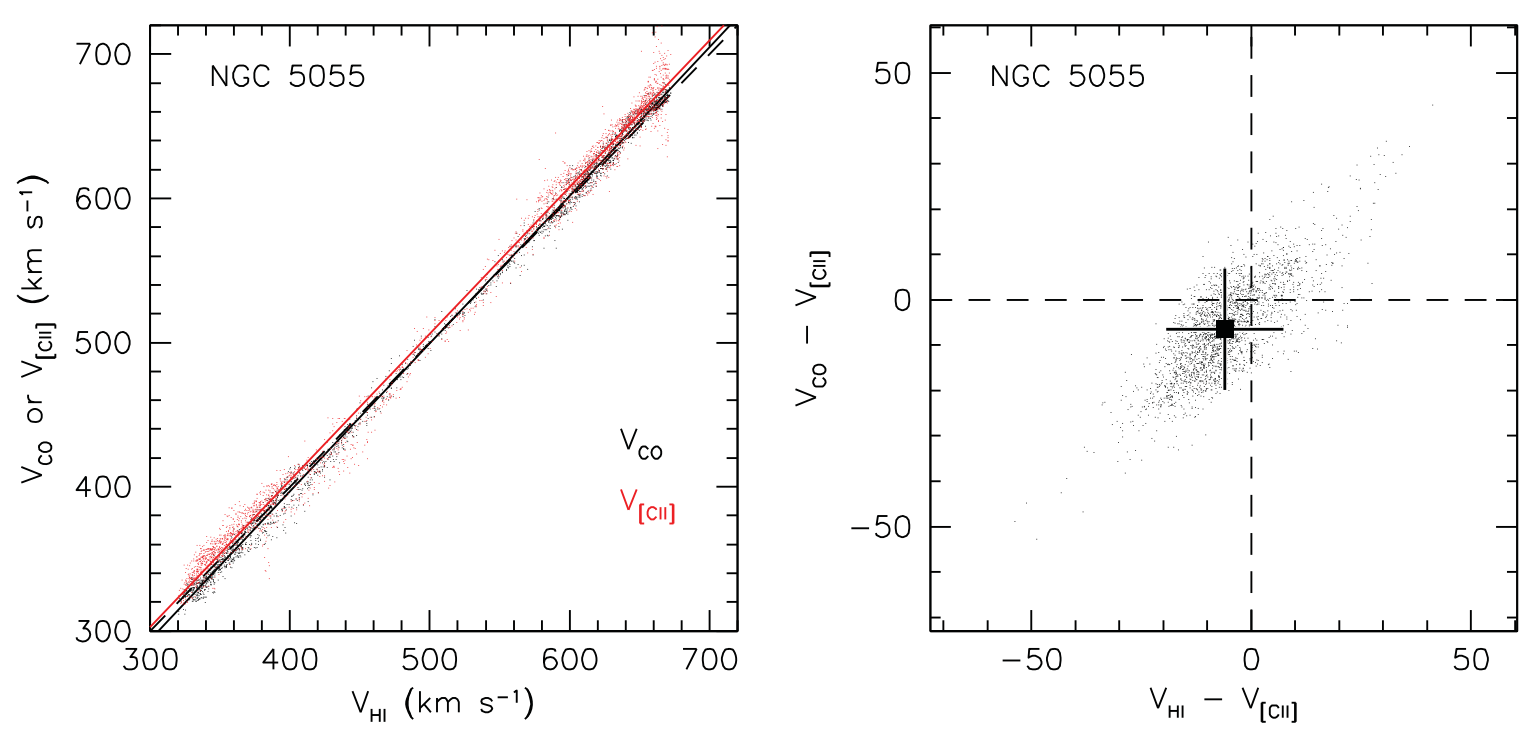

Figure 2. Pixel-by-pixel comparison of the [C II], H I, and CO velocity field values for NGC 5055. Left: CO (black) and [C II] (red) velocities plotted against the $\mathrm{H}$ I velocities. The black dashed line is the line of equality. The red and black full lines are linear least-squares fits of the CO (black) and the [C II] (red) velocities. Right: differences between $\mathrm{H}_{\mathrm{I}}$ and $[\mathrm{C}$ II] velocities plotted against the differences between $\mathrm{CO}$ and $[\mathrm{C}$ II] velocities. The filled square indicates the mean value, the errorbars indicate the $1 \sigma$ spread in differences. There are small offsets, which we attribute to systematics in the determination of the [C II] velocities (see the text).

(The complete figure set (10 images) is available.)
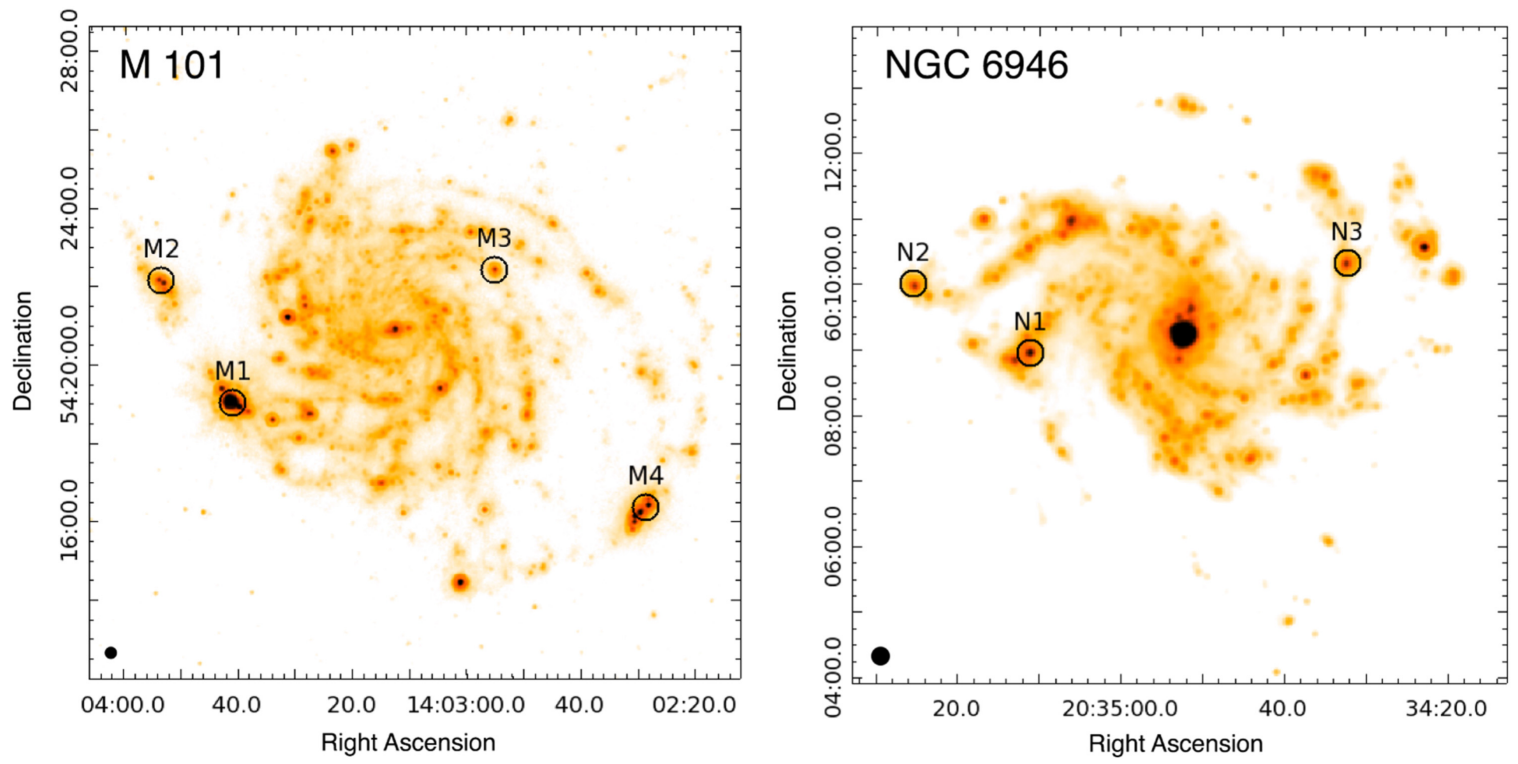

Figure 3. Positions of the four star-forming regions in M101 (left panel) and the three star-forming regions in NGC 6946 (right panel) that were observed with SOFIA overplotted on MIPS $24 \mu \mathrm{m}$ images.

\subsection{SOFIA [C II] Data}

Observations of a number of star-forming regions in M101 (NGC 5457) and NGC 6946 (PI Herrera-Camus, 02_0098) were carried out using the GREAT ${ }^{20}$ heterodyne receiver (Heyminck et al. 2012) on board SOFIA ${ }^{21}$ (Young et al. 2012). Figure 3 shows the positions of the star-forming regions observed overplotted on Spitzer MIPS $24 \mu \mathrm{m}$ maps.

\footnotetext{
${ }^{20}$ GREAT is a development by the MPI für Radioastronomie and the KOSMA/Universität zu Köln, in cooperation with the MPI für Sonnensystemforschung and the DLR Institut für Planetenforschung.

${ }^{21}$ SOFIA is jointly operated by the Universities Space Research Association, Inc. (USRA), under NASA contract NAS2-97001, and the Deutsches SOFIA Institut (DSI) under DLR contract 50 OK 0901 to the University of Stuttgart.
}

The four star-forming regions in M101 (M1, M2, M3, and M4) were observed on 2014 May 20 on a $\sim 1.2 \mathrm{hr}$ leg at an altitude of $45,000 \mathrm{ft}$. The measured system temperature was $\sim 3100 \mathrm{~K}$ for $[\mathrm{C}$ II] $158 \mu \mathrm{m}$ and on-source the integration times on M1, M2, M3, and M4 were 5.6, 6.3, 3.2, and 6.3 minutes, respectively. These data were obtained in beam switching mode, with a chopper throw of $\lesssim 50^{\prime \prime}$.

The three star-forming regions in NGC 6946 (N1, N2, and N3) were observed on 2014 May 21 on a $\sim 1.2 \mathrm{hr}$ leg at an altitude of $39,000 \mathrm{ft}$. The measured system temperature was $\sim 3200 \mathrm{~K}$ for $[\mathrm{C}$ II] $158 \mu \mathrm{m}$ and the on-source integration times on N1, N2, and N3 were 6.8, 6.3, and 6.3 minute, respectively. These observations used beam switching with amplitudes of $120^{\prime \prime}$ and $135^{\prime \prime}$. 
GREAT was set to its L1:L2 configuration, with L1 tuned to the [N II] $205 \mu \mathrm{m}$ transition $(1461.13 \mathrm{GHz})$ in the lower sideband and the L2 channel tuned to the [C II] $158 \mu \mathrm{m}$ transition $(1900.53 \mathrm{GHz})$ in the upper sideband. The half power beam width determined during the flight was 14 !. 1 at $1.89 \mathrm{THz}$ and $19 . ! 9$ at $1.34 \mathrm{THz}$. The spectra were analyzed in CLASS $^{22}$ and calibrated for a main beam coupling efficiency 0.70 for the L1 channel and 0.65 for the L2 channel (numbers provided by the GREAT project). The GREAT [C II] spectra were smoothed to a velocity resolution of $2.88 \mathrm{~km} \mathrm{~s}^{-1}$.

\section{RADIAL SURFACE DENSITY PROFILES}

We investigate the radial distribution of the [C II] from the Herschel observations, and compare to that of $\mathrm{H}$ I and $\mathrm{CO}$. We use the integrated intensity (zeroth moment) maps for this. As noted above, we have masked the $\mathrm{H}$ r and $\mathrm{CO}$ data so that only regions where $[\mathrm{C} \mathrm{II}$ ] observations exist are considered in this comparison. An example of these integrated intensity maps is shown in Figure 1 (left panel). The full collection of maps is available online.

To derive the radial profiles, we adopt the orientation parameters listed in Walter et al. (2008) (see also Table 1). We adopt a fixed (deprojected) ring width of $15^{\prime \prime}$ for all galaxies and tracers, implying that all values presented here are independent. The profiles are corrected for inclination, and we use the conversion factors listed in Leroy et al. (2008) to convert the $\mathrm{H}$ i and $\mathrm{CO}$ fluxes to column densities. We convert the inclination-corrected $[\mathrm{C}$ II] values from their original units of $\mathrm{W} \mathrm{m}{ }^{-2} \mathrm{sr}^{-1}$ into surface brightness units of $L_{\odot} \mathrm{pc}^{-2}$.

Figure 4 show the azimuthally averaged profiles for our sample galaxies. The errorbars show the rms spread in intensity values along each ring. These figures show that for the majority of cases the $\mathrm{H}$ I radial profile remains approximately constant with radius over the radial range covered here, while those of the $\mathrm{CO}$ and $[\mathrm{C}$ II] decline. The $[\mathrm{C}$ II] radial distribution therefore follows that of the $\mathrm{CO}$ more closely than that of the $\mathrm{H}$. We illustrate this further by overplotting the radial profiles for each tracer (expressed in terms of $R_{25}$; Walter et al. 2008 and Table 1), as shown in Figure 5 (left panel). To quantify the slope for each tracer we make (unweighted) exponential fits of the form $e^{-r / h}$ to each of the three sets of combined profiles. As expected, the slope of the average Hiprofile is consistent with being flat, with an exponential scale length of $h=-8.4 R_{25}$ with large uncertainties in the $h$-value. The scale length values of the $\mathrm{CO}$ and $[\mathrm{C}$ II] tracer are better defined. For the $\mathrm{CO}$ we find $h=\left(0.22_{-0.01}^{+0.03}\right) R_{25}$, which is consistent with the value found by Schruba et al. (2011) of $h=0.2 R_{25}$ for a partially different subsample of the HERACLES galaxies. For the $\left[\mathrm{C}\right.$ II] we find $h=\left(0.38_{-0.02}^{+0.03}\right) R_{25}$. For comparison, a purely exponential stellar Freeman disk (Freeman 1970) has a scale length $h=0.31 R_{25}$. The [C II] distribution is therefore slightly less compact than the $\mathrm{CO}$ one, and comparable with that of the optical component.

To confirm that this behavior applies to the entire disk, and is not dominated by, e.g., the bright inner parts, we also determine the exponential scale lengths for the radial ranges $R<0.5 R_{25}$ and $R>0.5 R_{25}$ separately. For $R<0.5 R_{25}$ we find that the scale lengths for $\mathrm{HI}, \mathrm{CO}$ and $[\mathrm{C} \mathrm{II}]$ are $h=\left(-0.41_{-0.08}^{+0.06}\right.$, $\left.0.19_{-0.02}^{+0.03}, 0.35_{-0.05}^{+0.08}\right)$, respectively. For the radii $R>0.5 R_{25}$, the respective values are $h=\left(1.24_{-0.38}^{+0.98}, 0.28_{-0.07}^{+0.17}, 0.45_{-0.06}^{+0.09}\right)$. In these two radial ranges we therefore see the same trends as

\footnotetext{
${ }^{22}$ http://www.iram.fr/IRAMFR/GILDAS/
}

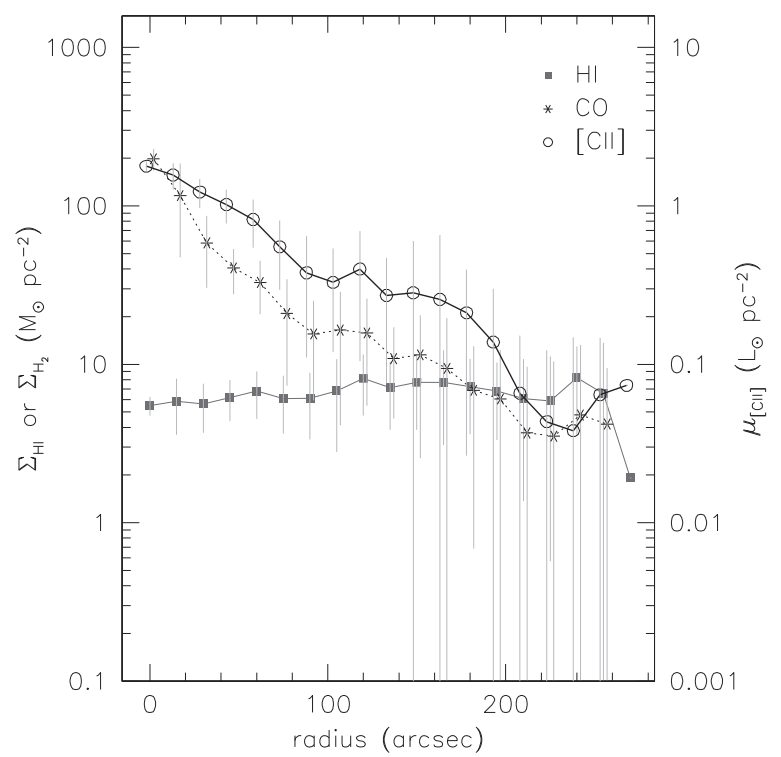

Figure 4. $\mathrm{CO}\left(\mathrm{H}_{2}\right), \mathrm{H}_{\mathrm{I}}$, and $[\mathrm{C} \mathrm{II}]$ radial profiles for NGC 5055 over the area covered by the $[\mathrm{C}$ II] observations. Filled squares show the $\mathrm{H}$ i surface density in $M_{\odot} \mathrm{pc}^{-2}$. The $\mathrm{H}_{2}$ surface density in $M_{\odot} \mathrm{pc}^{-2}$ is shown with the star symbols. The [C II] surface brightness in $L_{\odot} \mathrm{pc}^{-2}$ is shown with open circles. For clarity, the $\mathrm{CO}$ and $[\mathrm{C} \mathrm{II}]$ profiles have been offset by $2^{\prime \prime}$ and $-2^{\prime \prime}$ with respect to the $\mathrm{H}$ i points, respectively. The errorbars indicate the rms spread in values found along each ring. All surface densities and brightnesses have been corrected for inclination.

(The complete figure set (10 images) is available.)

over the entire disk, showing that the behavior seen is systematic.

We quantify this further by plotting the ratios of the surface densities, $\mathrm{HI}_{\mathrm{I}} /[\mathrm{C} \mathrm{II}]$ and $\mathrm{CO} /[\mathrm{C} \mathrm{II}]$, in Figure 5 (right panel), where we again express the radius in terms of the optical radius, $R_{25}$. We also show the average value and rms spread for all galaxies combined and determined in bins of width $0.2 R_{25}$.

The $\mathrm{CO} /[\mathrm{C}$ II $]$ ratio declines by a factor $\sim 2.8$ between the center and $R_{25}$. This implies again that the [C II] is somewhat less concentrated than the $\mathrm{CO}$ emission. On the other hand, the $\mathrm{H}$ I/[C II $]$ ratio increases by a factor of $\sim 9.8$, consistent with a much "flatter" radial $\mathrm{H}_{\mathrm{I}}$ distribution. We conclude that the [C II] follows the $\mathrm{CO}$ more closely than the $\mathrm{H}$ I and note that this is consistent with the finding that both $\mathrm{CO}$ and $[\mathrm{C}$ II] are approximately linear tracers of the SFR surface density, whereas $\mathrm{H}_{\mathrm{I}}$ is not (e.g., Bigiel et al. 2008; Leroy et al. 2008, 2013; Herrera-Camus et al. 2015).

\section{VELOCITIES}

While pixel-by-pixel comparisons are a good way to show that velocities agree with each other locally, these kind of data will not be available for the majority of galaxies studied at high redshift. For many galaxies only an integrated spectrum (global profile) will be available.

Using the $\mathrm{HI}, \mathrm{CO}$ and $[\mathrm{C} \mathrm{II}]$ data cubes we determine integrated spectra of our sample galaxies by adding the total flux in each velocity channel, using only the areas where $[\mathrm{C}$ II] observations were done. As these regions cover the major axes of the galaxies, the global profiles should contain most of the rotation signal of the sample galaxies. The profiles are shown in Figure 6. Due to the large difference in velocity resolution 

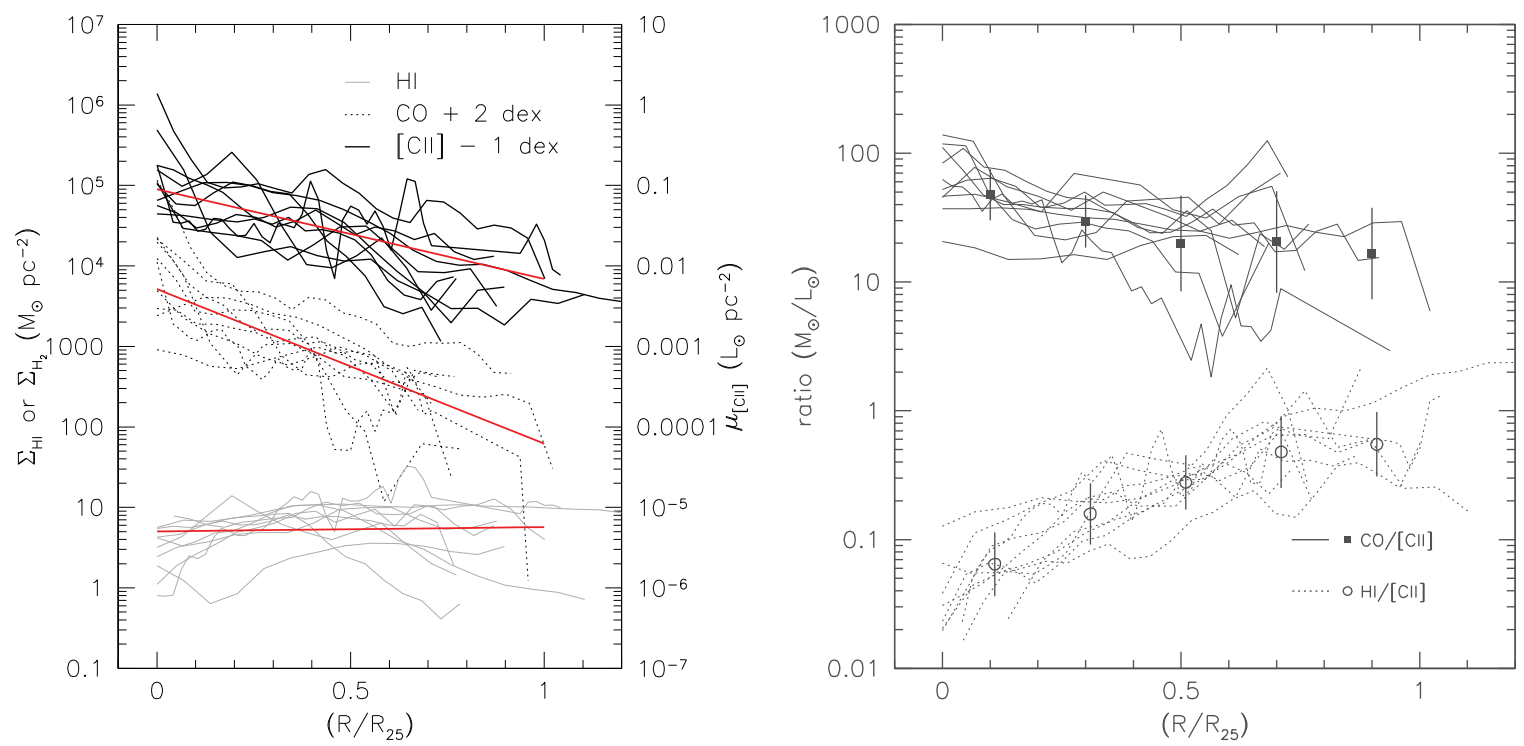

Figure 5. Left: radial profiles of the $\mathrm{H}_{\mathrm{I}}$ (thin gray lines), $\mathrm{CO}$ (dotted black lines), and [C II] (thick black lines) as shown in Figure 4 . For clarity the $\mathrm{CO}$ profiles have been offset vertically by +2 dex, the [C II] profiles by -1 dex. The red lines show the unweighted exponential fits to the combined profiles of each tracer. Right: ratios of the $\mathrm{CO}, \mathrm{H}$ I, and $[\mathrm{C} \mathrm{II}]$ radial profiles shown in Figure 4. Full curves show the $\mathrm{CO} /[\mathrm{C} \mathrm{II}]$ surface density ratio as a function of radius for the individual galaxies. The dotted curves show the same for the $\mathrm{H}_{\mathrm{I}} /\left[\mathrm{C} \mathrm{II}\right.$ surface density ratios. The radius is expressed in terms of the optical radius $R_{25}$. Also shown are the average values and rms spread in bins of $0.2 R_{25}$. Filled squares show the $\mathrm{CO} /[\mathrm{C}$ II] averages, open circles the $\mathrm{H} \mathrm{I} /[\mathrm{C} \mathrm{II}]$ values. The ratios on the vertical axis are derived from the original units in Figure 4. The $\mathrm{H}_{\mathrm{I}} /[\mathrm{C}$ II] data have been shifted down by 2 dex for clarity.
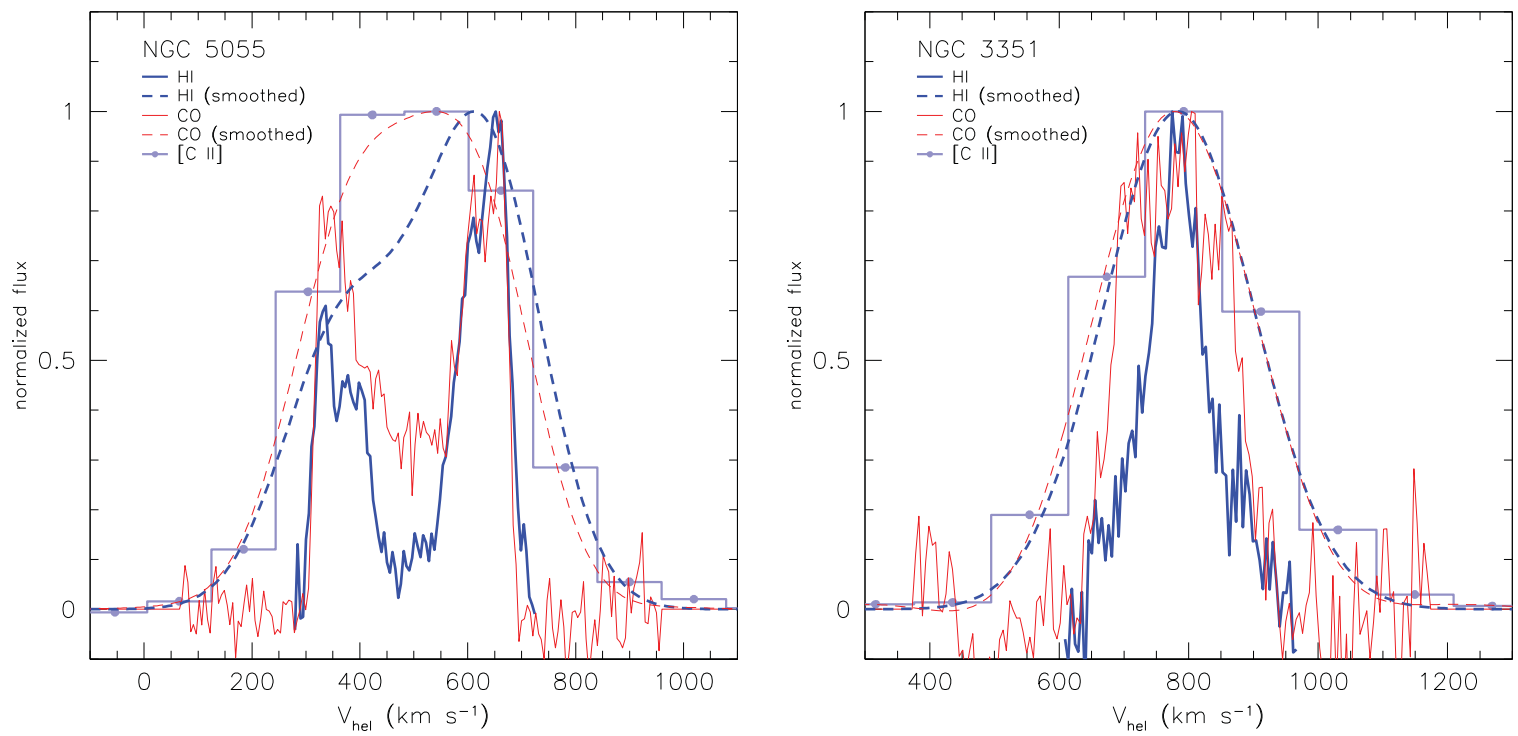

Figure 6. Global profiles as measured in $\mathrm{CO},[\mathrm{C}$ II], and $\mathrm{H}$. Global profiles are all derived from the masked "strip" cubes. The thick, blue full line shows the $\mathrm{H}$ I profile, the thin red full line the $\mathrm{CO}$ profile. The light-blue histogram with superimposed filled circles shows the [C II] global profile. The thick blue and thin red dashed lines show the $\mathrm{H}$ a and CO global profiles, respectively, both convolved to the velocity resolution of $239 \mathrm{~km} \mathrm{~s}^{-1}$ of the [C II] data. Left panel shows NGC 5055, right panel shows NGC 3351.

(The complete figure set (10 images) is available.)

between the $\left[\mathrm{C}\right.$ II] data $\left(239 \mathrm{~km} \mathrm{~s}^{-1}\right)$ on the one hand and $\mathrm{H} \mathrm{I}$ and $\mathrm{CO}$ data on the other hand $\left(2.6\right.$ or $\left.5.2 \mathrm{~km} \mathrm{~s}^{-1}\right)$, we show the $\mathrm{H}$ I and $\mathrm{CO}$ profiles both at their native velocity resolution (full curves) and also convolved in velocity (dashed curves, using a Gaussian convolution function) to the $\left[\mathrm{C}_{\mathrm{II}}\right]$ resolution.

The coarse velocity resolution of the $\left[\mathrm{C}_{\mathrm{II}}\right]$ data in combination with the velocity width of the galaxies means that for many of the galaxies the [C II] data do not allow us to resolve the double horn profile that is typically seen in $\mathrm{H}$ I and $\mathrm{CO}$ (see Walter et al. 2008; Frank et al. 2015). Figure 6 shows one galaxy (NGC 5055, left panel) where the velocity width of the galaxy as measured in $[\mathrm{C}$ II] is significantly (a factor of $\sim 2$ ) larger than the velocity resolution of the $\left[\mathrm{C}_{\mathrm{II}}\right]$ data. Despite the coarse velocity resolution, it is still possible to directly compare features in the $[\mathrm{C} \mathrm{II}]$ spectrum with those in the convolved $\mathrm{CO}$ and $\mathrm{H}$ i spectra. We see that for this galaxy, the [C II] spectrum follows the convolved $\mathrm{CO}$ spectrum more closely than the convolved $\mathrm{H}$ I one. Also shown in that Figure is a more typical case (NGC 3351, right) where the [C II] velocity width is comparable to the $[\mathrm{C}$ II] velocity resolution. Here, no distinctive 
features can be made out in the spectra. This lack of features also applies to the remaining galaxies.

A global profile, or integrated spectrum, is determined by both the galaxy rotation curve and the surface density profile of the tracer (e.g., $\mathrm{CO}, \mathrm{H}_{\mathrm{I}},\left[\mathrm{C}_{\mathrm{II}}\right]$ ) used to measure the velocities. As all three gas tracers are embedded in the same mass potential, the measured rotation curve will to a very large degree only depend on the total mass density as a function of radius. Any difference between integrated spectra of the same galaxy, can therefore be directly attributed to differences in the radial distribution of the tracer used (see de Blok \& Walter 2014 for an extensive discussion). With none of the galaxies having a [C II] radial distribution significantly different from the $\mathrm{CO}$ distribution, we expect spectrally resolved [C II] global profiles to look more like the $\mathrm{CO}$ global profiles than their $\mathrm{H}$ I counterparts.

\section{SMALL-SCALE COMPARISONS}

In the previous sections we established that the $[\mathrm{C}$ II] and $\mathrm{CO}$ radial distributions are more similar to each other than to the $\mathrm{H}$ I distribution. This raises the question whether the $\mathrm{CO}$ and $[\mathrm{C}$ II] emission also originates from similar volumes in the galaxies. One possible way of studying this is by looking at the velocity dispersions of the components, which are an indication of the extent of the distribution along the line of sight.

We use the highly resolved SOFIA data obtained for starforming regions in M101 (NGC 5457) and NGC 6946. Figures 7 and 8 compare the $[\mathrm{C} \mathrm{II}], \mathrm{CO}$ and $\mathrm{H}$ ispectra at the positions of these star-forming regions. It is clear that $[\mathrm{C} \mathrm{II}]$ is only found at the velocities where $\mathrm{H}$ I and/or $\mathrm{CO}$ also occur. Note that the spatial resolution of the SOFIA observations is $\sim 0.5 \mathrm{kpc}$, so the individual pointings are probing a significant volume.

The majority of the profiles are approximately Gaussian, and we characterize them as such. The parameters of the fits are given in Table 2. In Figure 9 we compare the fitted velocity widths (Gaussian dispersions) of the various profiles. We have excluded region $\mathrm{M} 3$ due to the low signal-to-noise of the $\left[\mathrm{C}_{\mathrm{II}}\right]$. The $[\mathrm{C}$ II] linewidths are intermediate between those of $\mathrm{CO}$ and $\mathrm{HI}$, but always closer to those of the CO. For the regions probed here, the agreement in $\mathrm{CO}$ and $[\mathrm{C}$ II] velocity dispersion values suggests that the distribution of $\mathrm{CO}$ and $[\mathrm{C} \mathrm{II}]$ along the line of sight is similar.

It also suggests that the contribution from photo-dissociation regions dominates the emission, at least in these regions. This implies that at least the bright $[\mathrm{C}$ II] emission is associated with $\mathrm{CO}$. This is a very similar situation to what we found for the integrated spectra above (see also Mookerjea et al. 2011, 2016; Braine et al. 2012; Okada et al. 2015).

\section{DISCUSSION AND SUMMARY}

We have shown that for our sample of nearby disk galaxies, the $\left[\mathrm{C}_{\mathrm{II}}\right]$ radial surface density distribution follows that of the $\mathrm{CO}$ more closely than that of the $\mathrm{H}$ I. For one galaxy (NGC 5055) we find that, at common spectral resolution, the $[\mathrm{C}$ II] integrated spectrum resembles that of the $\mathrm{CO}$ more than that of the $\mathrm{H}$.

We note that it is not a surprise that the $[\mathrm{C}$ II] follows the $\mathrm{CO}$ more closely than the H I: Herrera-Camus et al. (2015) show that for the KINGFISH galaxies the [C II] surface brightness is a good tracer of the SFR surface density. With the CO surface density also being a good tracer (Leroy et al. 2008), a tight

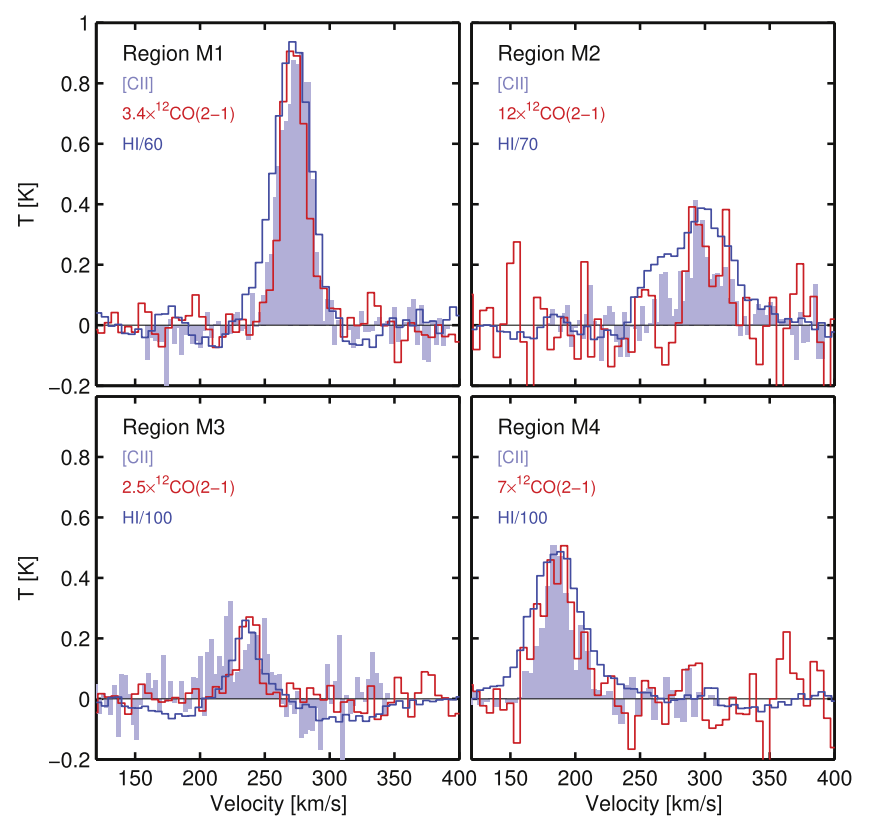

Figure 7. [C II], $\mathrm{H} \mathrm{I}_{\mathrm{I}}$ and $\mathrm{CO}$ spectra of the four star-forming regions in M101. The $\mathrm{H}$ i and $\mathrm{CO}$ peak intensities are scaled to approximately match the $[\mathrm{C}$ II] intensity. Positions of the regions are indicated in Figure 3.

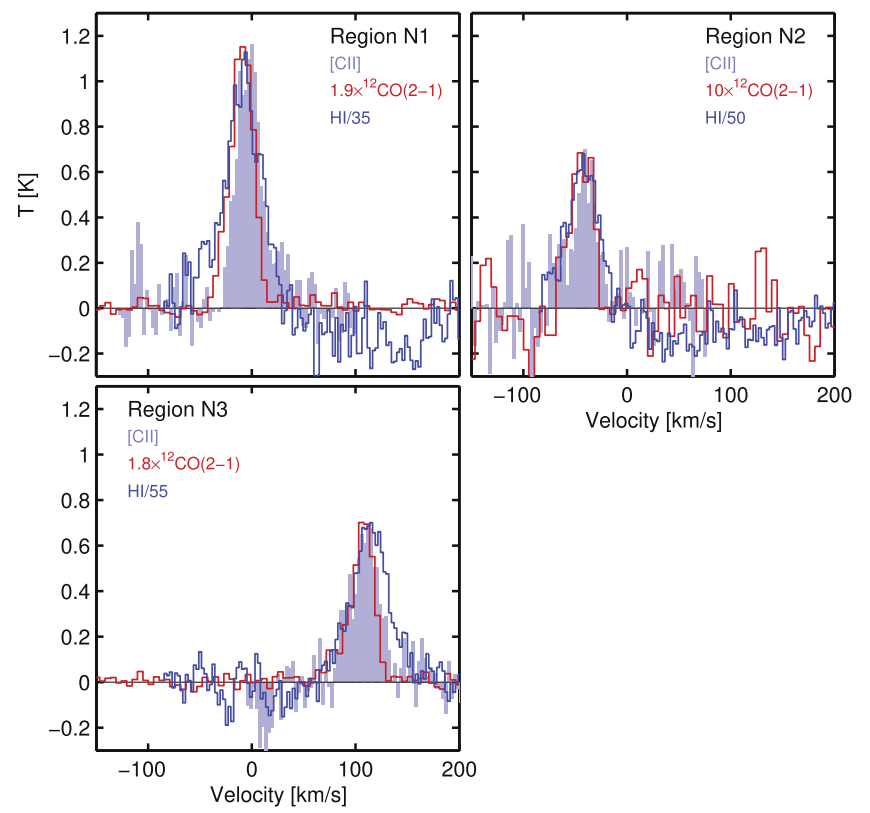

Figure 8. [C II], $\mathrm{H}$ I, and $\mathrm{CO}$ spectra of the three star-forming regions in NGC 6946. The $\mathrm{H}$ i and $\mathrm{CO}$ peak intensities are scaled to approximately match the $[\mathrm{C}$ II] intensity. Positions of the regions are indicated in Figure 3.

relation between $\mathrm{CO}$ and $[\mathrm{C} \mathrm{II}]$ is therefore expected (on the scales considered here). This correspondence is also found on the scales of individual star-forming regions, as shown by the SOFIA [C II] data presented here. We note that these conclusions may not be applicable for very low metallicity objects, where $\mathrm{CO}$ can be deficient, or ultra-luminous infra-red galaxies, where a [C $\mathrm{II}]$ deficit has been observed, as already noted in the introduction.

The physical origins of the tight relation between $\mathrm{CO}$ and $[\mathrm{C} \mathrm{II}]$ are not entirely clear. $[\mathrm{C} \mathrm{II}]$ emission can originate from both the ionized and the neutral phase of the ISM. While 
Table 2

Gaussian Fit Parameters

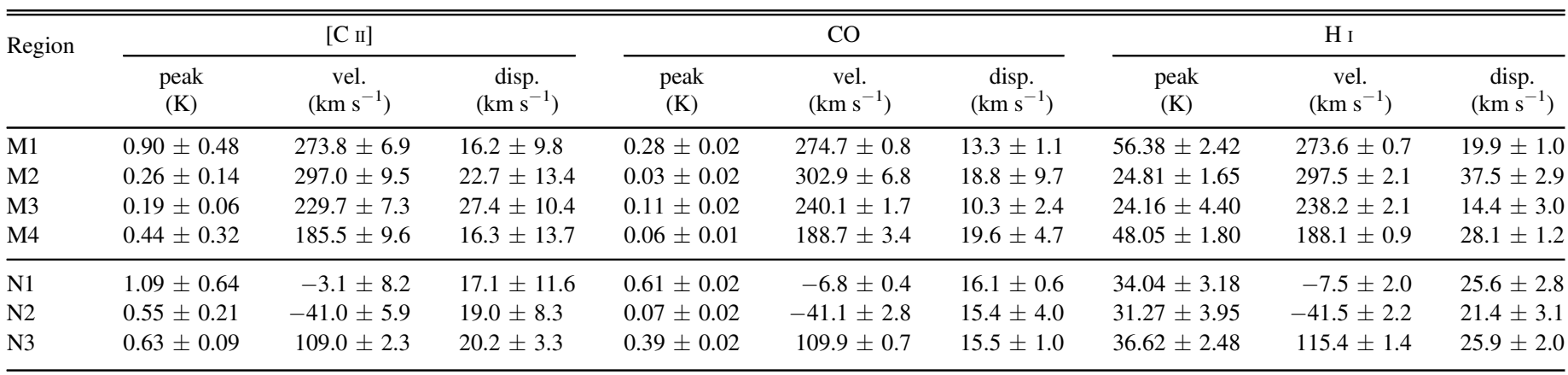

Note. Gaussian fit parameters of the profiles shown in Figures 7 and 8. "peak," "vel.," and "disp." indicate the peak flux, central velocity and velocity dispersion, respectively.

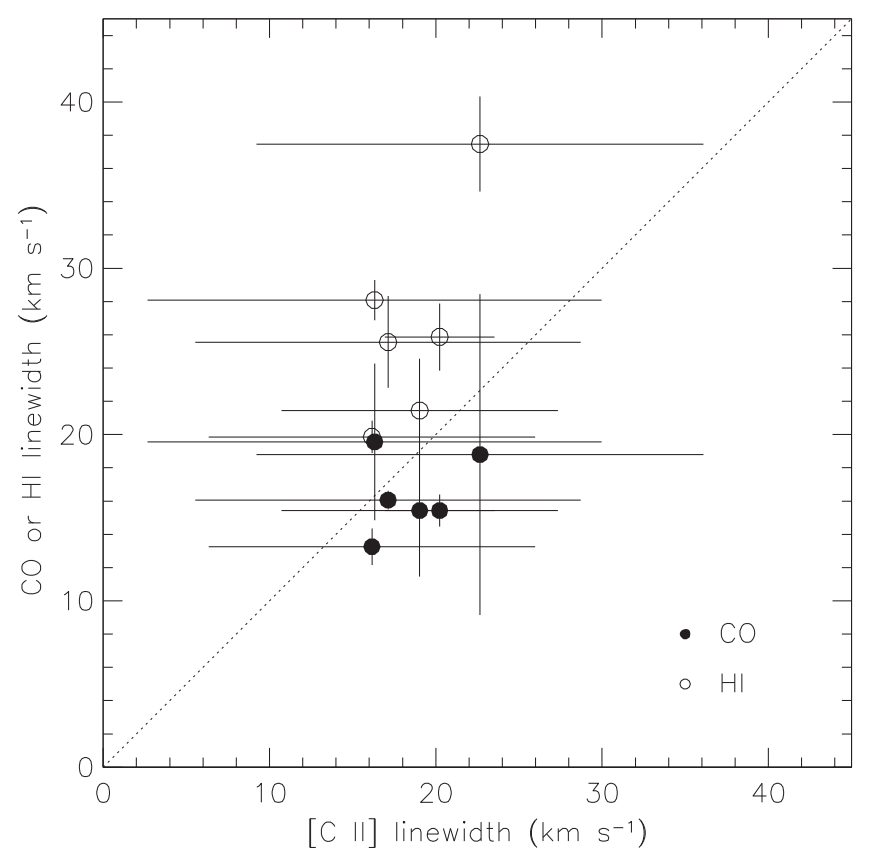

Figure 9. Comparison of the $[\mathrm{C} \mathrm{II}], \mathrm{H} \mathrm{I}$, and $\mathrm{CO}$ linewidths (Gaussian dispersions) of the spectra shown in Figures 7 and 8. Filled circles compare the $\mathrm{CO}$ and $\left[\mathrm{C} \mathrm{II}\right.$ linewidths, open circles compare the $\mathrm{H}_{\mathrm{I}}$ and the $[\mathrm{C} \mathrm{II}$. Errorbars indicate $95 \%$ confidence intervals. Region M3 is not shown due to the low signal-to-noise of the $[\mathrm{C}$ II] spectrum. Note that the $\mathrm{CO}$ values linewidths agree better with the [C II] values than the $\mathrm{H}_{\mathrm{I}}$ linewidths do.

naively one would expect that the good correlation with SFR suggests that the majority of the $[\mathrm{C}$ II] emission arises from the ionized phase, there is no evidence for the presence of a very significant ionized component in most galaxies. The ionized gas contribution of $[\mathrm{C}$ II] can be traced reliably by the [N $\mathrm{II}]$ $205 \mu \mathrm{m}$ line, which has a very similar critical density. This fraction has been found to be relatively small in nearby galaxies ( $30 \%)$, K. V. Croxall et al. 2016, in preparation). Therefore, at least in nearby galaxies, it is more likely that the [C II] emission is dominated by the contribution of the neutral gas, in which case the observed relation is due to the heating of the neutral gas by $6-13 \mathrm{eV}$ photons which are a reasonable tracer of the instantaneous SFR. An extensive and more complete discussion of the origins of the $[\mathrm{C} \mathrm{II}]$ emission is given in K. V. Croxall et al. (2016, in preparation).
We conclude that if spatially unresolved [C II] observations are used to determine dynamical masses then the wellcharacterized length scale of the $\mathrm{CO}$ is preferred over that of the H I (see Schruba et al. 2011; de Blok \& Walter 2014) and approximates the dynamical mass that would have been derived from the $[\mathrm{C}$ II] itself to within a factor of two. The $\mathrm{CO}$ scale length is known to be similar to that of the stellar disk in nearby galaxies (Young et al. 1995; Regan et al. 2001; Schruba et al. 2011) and maybe even in high- $z$ main sequence galaxies (Bolatto et al. 2015).

Our conclusions can be applied to galaxies observed at high redshift, provided they are dominated by rotation and assuming that the locally observed relations between $[\mathrm{C}$ II], $\mathrm{CO}$ and SFR hold there as well.

W.J.G.d.B. was supported by the European Commission (grant FP7-PEOPLE-2012-CIG \#333939). Beyond the Peak research has been supported by a NASA/JPL grant (RSA 1427378). J.D.S. gratefully acknowledges visiting support from the Alexander von Humboldt Foundation and the Max Planck Institute für Astronomie. A.D.B. and R.H.C. acknowledge support from grants USRA-SOF020098, NSFAST0955836, and NSF-AST1412419, as well as visiting support from the Humboldt Foundation. This work is based in part on observations made with the NASA/DLR Stratospheric Observatory for Infrared Astronomy (SOFIA). Financial support for this work was provided by NASA.

\section{REFERENCES}

Bigiel, F., Leroy, A., Walter, F., et al. 2008, AJ, 136, 2846

Blyth, S., van der Hulst, T. M., Verheijen, M. A. W., et al. 2015, in Proc. of Advancing Astrophysics with the Square Kilometre Array (AASKA14), 128 Bolatto, A. D., Warren, S. R., Leroy, A. K., et al. 2015, arXiv:1507.05652 Braine, J., Gratier, P., Kramer, C., et al. 2012, A\&A, 544, A55

Brisbin, D., Ferkinhoff, C., Nikola, T., et al. 2015, ApJ, 799, 13 Carilli, C. L., \& Walter, F. 2013, ARA\&A, 51, 105

Colombo, D., Meidt, S. E., Schinnerer, E., et al. 2014, ApJ, 784, 4

Cormier, D., Madden, S. C., Lebouteiller, V., et al. 2015, A\&A, 578, A53

Croxall, K. V., Smith, J. D., Brandl, B. R., et al. 2013, ApJ, 777, 96

da Cunha, E., Groves, B., Walter, F., et al. 2013, ApJ, 766, 13

de Blok, W. J. G., \& Walter, F. 2014, AJ, 147, 96

de Blok, W. J. G., Walter, F., Brinks, E., et al. 2008, AJ, 136, 2648

Dickey, J. M., \& Kazes, I. 1992, ApJ, 393, 530

Förster Schreiber, N. M., Genzel, R., Lehnert, M. D., et al. 2006, ApJ, 645, 1062 Frank, B. S., de Blok, W. J. G., Walter, F., Leroy, A., \& Carignan, C. 2015, AJ, 151, 94

Freeman, K. C. 1970, ApJ, 160, 811 
Genzel, R., Tacconi, L. J., Combes, F., et al. 2012, ApJ, 746, 69

Genzel, R., Tacconi, L. J., Eisenhauer, F., et al. 2006, Natur, 442, 786

Herrera-Camus, R., Bolatto, A. D., Wolfire, M. G., et al. 2015, ApJ, 800, 1

Heyminck, S., Graf, U. U., Güsten, R., et al. 2012, A\&A, 542, L1

Kennicutt, R. C., Calzetti, D., Aniano, G., et al. 2011, PASP, 123, 1347

Kennicutt, R. C., Jr., Armus, L., Bendo, G., et al. 2003, PASP, 115, 928

Leroy, A. K., Walter, F., Bigiel, F., et al. 2009, AJ, 137, 4670

Leroy, A. K., Walter, F., Brinks, E., et al. 2008, AJ, 136, 2782

Leroy, A. K., Walter, F., Sandstrom, K., et al. 2013, AJ, 146, 19

Luhman, M. L., Satyapal, S., Fischer, J., et al. 2003, ApJ, 594, 758

Madden, S. C., Galametz, M., Cormier, D., et al. 2011, in EAS Publications Ser., 48,139

Maiolino, R., Cox, P., Caselli, P., et al. 2005, A\&A, 440, L51

Mathewson, D. S., Ford, V. L., \& Buchhorn, M. 1992, ApJS, 81, 413

Mookerjea, B., Israel, F., Kramer, C., et al. 2016, A\&A, 586, A37

Mookerjea, B., Kramer, C., Buchbender, C., et al. 2011, A\&A, 532, A152

Okada, Y., Requena-Torres, M. A., Güsten, R., et al. 2015, A\&A, 580, A54

Pilbratt, G. L., Riedinger, J. R., Passvogel, T., et al. 2010, A\&A, 518, L1
Pineda, J. L., Langer, W. D., Velusamy, T., \& Goldsmith, P. F. 2013, A\&A, 554, A103

Poglitsch, A., Waelkens, C., Geis, N., et al. 2010, A\&A, 518, L2

Regan, M. W., Thornley, M. D., Helfer, T. T., et al. 2001, ApJ, 561, 218

Regan, M. W., Thornley, M. D., Vogel, S. N., et al. 2006, ApJ, 652, 1112

Schoniger, F., \& Sofue, Y. 1994, A\&A, 283, 21

Schruba, A., Leroy, A. K., Walter, F., et al. 2011, AJ, 142, 37

Schruba, A., Leroy, A. K., Walter, F., et al. 2012, AJ, 143, 138

Smith, J. D. T., Draine, B. T., Dale, D. A., et al. 2007, ApJ, 656, 770

Sofue, Y. 1992, PASJ, 44, L231

Tully, R. B., \& Fisher, J. R. 1977, A\&A, 54, 661

Venemans, B. P., McMahon, R. G., Walter, F., et al. 2012, ApJL, 751, L25

Verheijen, M., van Gorkom, J. H., Szomoru, A., et al. 2007, ApJL, 668, L9

Vogt, N. P., Forbes, D. A., Phillips, A. C., et al. 1996, ApJL, 465, L15

Walter, F., Brinks, E., de Blok, W. J. G., et al. 2008, AJ, 136, 2563

Walter, F., Decarli, R., Carilli, C., et al. 2012, ApJ, 752, 93

Wilson, C. D. 1995, ApJL, 448, L97

Young, E. T., Becklin, E. E., Marcum, P. M., et al. 2012, ApJL, 749, L17

Young, J. S., Xie, S., Tacconi, L., et al. 1995, ApJS, 98, 219 